\title{
Testing collapse models with levitated nanoparticles: Detection challenge
}

\author{
A. Vinante,,${ }^{1, *}$ A. Pontin,,${ }^{2, \dagger}$ M. Rashid, ${ }^{1}$ M. Toroš ${ }^{2}$ P. F. Barker,${ }^{2}$ and H. Ulbricht ${ }^{1}$ \\ ${ }^{1}$ Department of Physics and Astronomy, University of Southampton, Southampton SO17 1BJ, England, United Kingdom \\ ${ }^{2}$ Department of Physics and Astronomy, University College London, London WC1E 6BT, England, United Kingdom
}

(Received 20 March 2019; published 16 July 2019)

\begin{abstract}
We consider a nanoparticle levitated in a Paul trap in ultrahigh cryogenic vacuum, and look for the conditions which allow for a stringent noninterferometric test of spontaneous collapse models. In particular we compare different possible techniques to detect the particle motion. Key conditions which need to be achieved are extremely low residual pressure and the ability to detect the particle at ultralow power. We compare three different detection approaches based, respectively, on an optical cavity, an optical tweezer, and an electrical readout, and for each one we assess advantages, drawbacks, and technical challenges.
\end{abstract}

DOI: 10.1103/PhysRevA.100.012119

\section{INTRODUCTION}

Spontaneous wave-function collapse (or dynamical reduction) models (CMs) [1-5] have been proposed to reconcile the linear and deterministic evolution of quantum mechanics with the nonlinearity and stochasticity of the measurement process. According to CMs, random collapses in space (i.e., localizations) of the wave function of any system occur spontaneously, independently of measurement processes, leading to a progressive spatial localization. The collapse rate scales with the size of the system, leading to rapid localization of any macroscopic system, while giving no measurable effect at the microscopic level, where standard quantum mechanics holds. Importantly, CMs lead to a natural solution of the measurement problem, by predicting the emergence of welldefined outcomes in any experiment in agreement with the Born rule.

The most general model described in literature is the continuous spontaneous localization (CSL) model [2,3], a refined version of the earliest collapse model proposed by Ghirardi, Rimini, and Weber (GRW) [1]. Other more specific models have been proposed in literature, the most notable being the Diósi-Penrose gravity-induced collapse model [6-8]. In this paper we will focus on the CSL model only, as other models can be usually regarded as special cases, or slight variations, of CSL. CSL is characterized by two phenomenological constants, a collapse rate $\lambda$ and a characteristic length $r_{C}$, which characterize, respectively, the intensity and the spatial resolution of the spontaneous collapse process. $\lambda$ and $r_{C}$ are free parameters which should be derived, or bounded, by experiments. The standard conservative values suggested by GRW are $\lambda \simeq 10^{-16} \mathrm{~s}^{-1}$ and $r_{C}=10^{-7} \mathrm{~m}[1,2]$ and are sufficient to guarantee almost instantaneous localization of macroscopic objects. A strongly enhanced value for the collapse rate has been suggested by Adler [9], motivated by the requirement of making the wave-function collapse effective at the level

\footnotetext{
*A.Vinante@soton.ac.uk

†a.pontin@ucl.ac.uk
}

of latent image formation in the photographic process. The values of $\lambda$ suggested by Adler are $\approx 10^{9 \pm 2}$ times larger than the GRW values at $r_{C}=10^{-7} \mathrm{~m}$, and $\approx 10^{11 \pm 2}$ times larger at $r_{C}=10^{-6} \mathrm{~m}$.

Several precision experiments have been recently exploited to set significant bounds on the CSL model parameters. The direct effect of collapse models such as CSL is to suppress quantum superpositions, resulting in a loss of coherence in interferometric matter-wave experiments [10]. On top of that, the noise field associated with the collapse implies a violation of the energy conservation. So called noninterferometric tests have been proposed to look for these effects, which include spontaneous emission of $\mathrm{x}$ rays $[11,12]$, force noise in mechanical systems [13-24], and spontaneous heating of bulk matter [25] or ultracold atoms [26,27]. At present, noninterferometric tests set by far the strongest bound on CSL parameters, which are summarized in Fig. 1.

Here, we consider noninterferometric tests based on mechanical systems. In this approach, one looks for the universal force noise which is predicted to be induced by CSL in any massive mechanical system. So far, experiments based on ultracold cantilevers $[19,20]$ and gravitational wave detectors [21-24] are setting the strongest bounds. It has been suggested that levitated nanoparticles or microparticles would be a nearly ideal platform to perform more sensitive tests [28,29]. Ideally, one needs to work with the lowest possible temperature and dissipation in order to minimize thermal noise. We consider a possible experimental implementation based on ion trap techniques and point out that, for the extremely low thermal noise required by this experiment, the nanoparticle detection becomes a very crucial issue. We consider three possible detection techniques and discuss potential advantages and drawbacks.

\section{PHYSICAL SYSTEM AND THE BASIC MODEL}

\section{A. CSL noise}

Our goal is to monitor a levitated nanoparticle in order to detect or place strong upper bounds on the universal force noise predicted by spontaneous collapse models such as CSL. 


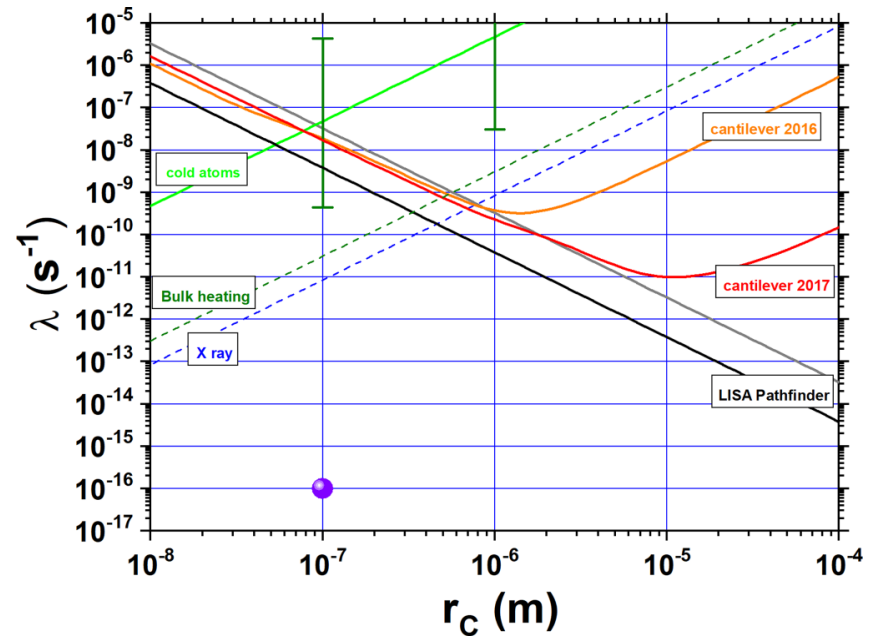

FIG. 1. Current upper limits on the CSL model collapse rate from different noninterferometric experiments. Solid lines refer to bounds from mechanical or cold atom experiments, and dashed lines refer to experimental bounds probing the CSL field at very high frequency, specifically, x-ray spontaneous emission (dashed blue) [12], bulk heating in solid matter (dashed dark green) [25], cold atoms (light green) [26], the cantilever 2016 experiment (orange) [19], the cantilever 2017 experiment (red) [20], LISA Pathfinder early data (gray) [21,22], and final data (black) [23]. The theoretical parameters suggested by Adler [9] and GRW [1] are also shown.

The CSL force noise power spectral density (PSD) acting on a homogeneous sphere can be written as [17-19]

$$
S_{f f, \mathrm{CSL}}=\frac{32 \pi^{2} \hbar^{2} \lambda r_{C}^{2} \rho^{2} R^{2}}{3 m_{0}^{2}}\left[1-\frac{2 r_{C}^{2}}{R^{2}}+e^{-\frac{R^{2}}{r_{C}^{2}}}\left(1+\frac{2 r_{C}^{2}}{R^{2}}\right)\right]
$$

where $\rho$ is the particle mass density, $R$ is the particle radius, and $m_{0}$ is the nucleon mass [30].

Note that Eq. (1) grows as $R^{2}$ for $R \gg r_{C}$, scaling therefore as a surface noise, and as $R^{6}$ for $R \ll r_{C}$. So, if the main background is surface force noise, as for instance due to gas collisions, the signal to noise ratio (SNR) is almost constant for $R>r_{C}$. If the main background force noise scales with the volume (as in the case of material-dependent losses) the SNR, according to Eq. (1), will feature a shallow maximum at $R \simeq 2 r_{C}$. Therefore, if we wish to probe the standard value $r_{C}=100 \mathrm{~nm}$, as a general rule the radius of the particle should be at least of the order of $200 \mathrm{~nm}$.

A similar analysis can be performed also for other collapse models. For example, for the Diósi-Penrose (DP) model the force noise power spectral density is given by $S_{f f, \mathrm{DP}}=$ $\frac{2 \sqrt{\pi}}{9} G \hbar R^{3} \rho^{2}\left(\frac{a}{r_{\mathrm{DP}}}\right)^{2}$, where $G$ is the gravitational constant, $R$ $(\rho)$ is the radius (density) of the nanoparticle, $a$ is the lattice constant of the material, $r_{\mathrm{DP}}$ is the DP cutoff parameter [31], and we have assumed $r_{\mathrm{DP}} \gg a[17,32]$. In particular, the results in the following sections can be reinterpreted in terms of the DP parameter $r_{\mathrm{DP}}$ by exploiting the relation $S_{f f, \mathrm{DP}}\left(r_{\mathrm{DP}}\right)=S_{f f, \mathrm{CSL}}\left(\lambda, r_{C}\right)$, i.e., one has a simple relation between $r_{\mathrm{DP}}$ and $\lambda\left(r_{c}\right)$ at a fixed value of $r_{c}(\lambda)$.

\section{B. Particle}

For simplicity we will consider a spherical nanoparticle, although comparable results could be achieved by other geometries [24]. We will take $\mathrm{SiO}_{2}$ as standard material. Although it is not the optimal choice for CSL, because of the relatively low density, it is the most used material when optical detection is involved. In general, optical detection requires low absorption dielectric materials, such as silicon or silica. In principle a much larger variety of materials could be used in the case of electrical detection.

\section{Paul trap}

As we wish to work in an ultracold and ultraisolated environment, standard optical trapping is not viable because of the strong heating of the nanoparticle. Alternative levitation methods which avoid this problem are Paul traps [33] and superconducting magnetic traps [34]. Both methods cause very small heat dissipation in the levitated particle, so they are expected to be compatible with a cryogenic environment.

We will assume here a Paul trap approach, as related technology has been pushed to quite an advanced level by the ion trapping community. For the sake of simplicity we will not go here into technical details on the geometry of the trap. We will only assume that the secular resonant frequency of one relevant translational mode can be set at $f_{0}=1 \mathrm{kHz}$. Such a value can be obtained by a Paul trap with electrode effective distance of the order of $1 \mathrm{~mm}$ or smaller, voltage bias of some tens of volts, and a charge on the particle of 10-1000 e. These parameters should be also readily compatible with a cryogenic operation.

Among possible sources of noise and decoherence related to the trap, we can mention surface losses in the electrodes and voltage noise in the driving ac and dc bias. Bias voltage noise is a known effect in ion traps, and can be a potentially limiting factor here because the trap potentials have to be kept continuously active. For an order-of-magnitude estimation, let us assume an electronics voltage noise $S_{v}=10 \mathrm{nV} / \sqrt{\mathrm{Hz}}$ at the secular frequency. This value corresponds to a dynamic range larger than $10^{9}$, and should be readily achievable, although it seems nontrivial to improve much over this value. Then, for a trapped charge of $q=30 e$, and an effective electrode distance of $500 \mu \mathrm{m}$, one estimates a force noise $S_{f} \simeq S_{v} q / d=$ $9.6 \times 10^{-23} \mathrm{~N} / \sqrt{\mathrm{Hz}}$. As shown in Fig. 3, this noise would be comparable with the effect predicted by the CSL model for the standard length parameter $r_{C}=10^{-7} \mathrm{~m}$ and the collapse rate $\lambda=10^{-10} \mathrm{~Hz}$. Therefore, a heavy suppression of electronic noise would be needed in order to probe the CSL collapse rate much lower than the latter value. In addition, we note that this noise contribution would increase for larger charge.

\section{Environment}

The environment of the particle leads to noise and decoherence through many different channels. A minimal list of sources includes scattering with gas particles and scattering, absorption, and emission of thermal photons. In addition, any trapping mechanism typically involves some kind of decoherence. For a Paul trap, besides voltage noise in the driving electrodes, there will be interaction with the electrode surface, 
as well as electrical losses if an electrical detection circuit is coupled to the trap. Ambient vibrational noise (seismic or acoustic noise) has to be eventually considered.

Collision with gas particles and emission of blackbody radiation will significantly affect the particle dynamics; not only do they represent a noise source but they are also the main mechanisms for the thermalization of the particle. Thus, the residual gas pressure $P_{g}$ and temperature $T_{g}$, together with the steady-state power $W_{\text {abs }}$ absorbed by the particle will determine its equilibrium bulk temperature $T$ as discussed in the following section.

\section{Thermal equilibrium}

The heat flow from a hot nanoparticle to a cold surrounding gas in the molecular regime can be calculated using the formula [35]

$$
\dot{Q}_{\text {gas }}=-\frac{\alpha \pi R^{2} P_{g} v_{t}}{2 T_{g}} \frac{\gamma_{s}+1}{\gamma_{s}-1}\left(T-T_{g}\right)
$$

where $\alpha$ is a thermal accommodation factor, $v_{t}=$ $\sqrt{8 k_{B} T_{g} /(\pi m)}$ is the gas thermal velocity with $m$ the molecular mass, and $\gamma_{s}$ is the specific-heat ratio. Here all parameters can be easily determined, except for the accommodation factor $0<\alpha<1$. Typical values of order 0.4 are reported in literature for specific experimental situations [35].

The heat flow by blackbody radiation is described by the expression [36]

$$
\dot{Q}_{\mathrm{BB}}=-\frac{72 \zeta(5)}{\pi^{2}} \frac{V k_{B}^{5}}{c^{3} \hbar^{4}} \operatorname{Im} \frac{\epsilon_{\mathrm{BB}}-1}{\epsilon_{\mathrm{BB}}+2}\left(T^{5}-T_{g}^{5}\right)
$$

where $V$ is the particle volume and $\zeta(5) \approx 1.04$ is the Riemann zeta function. The dependence on $T^{5}$ is typical of a subwavelength nanoparticle. Here, the flow is controlled by the absorption coefficient $\epsilon_{\mathrm{abs}}=\operatorname{Im}\left[\left(\epsilon_{\mathrm{BB}}-1\right) /\left(\epsilon_{\mathrm{BB}}+2\right)\right]$ where $\epsilon_{\mathrm{BB}}$ is the blackbody emissivity. For typical situations, as for instance silica at $100 \mathrm{~K}$, this term can be taken of the order of 0.1 .

By setting $\dot{Q}_{\mathrm{BB}}+\dot{Q}_{\mathrm{gas}}+W_{\mathrm{abs}}=0$ one can estimate the equilibrium internal temperature $T$ of the particle as a function of the environmental conditions and the input power. Figure 2 illustrates the dependence of the equilibrium temperature of the nanosphere on the gas pressure $P_{g}$ and absorbed power $W_{\mathrm{abs}}$ in a relevant region of the parameter space. Two regimes are clearly visible. For low pressure and high power the particle is thermalized by radiation, and the equilibrium temperature is independent of pressure. For high pressure and low power the particle is thermalized by the gas and the equilibrium temperature depends solely on the ratio $W_{\mathrm{abs}} / P_{g}$.

\section{Thermal force noise}

To estimate the thermal force noise due to the gas in a hot-particle scenario we follow the model of Ref. [37], i.e., we separately consider the contributions due to the impinging and emerging gas particles. The model assumes two different baths with temperature $T_{i}=T_{g}$ for the impinging molecules and $T_{e}=T_{i}+a\left(T-T_{i}\right)$ for the emerging molecules where $a$ is another phenomenological accommoda-

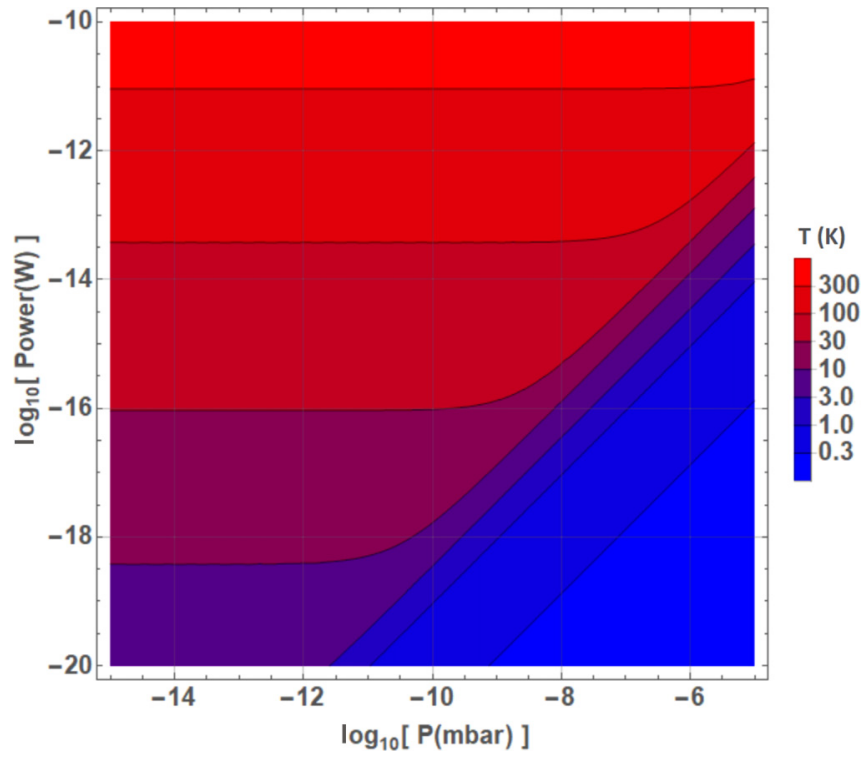

FIG. 2. Internal temperature of the nanosphere as a function of the gas pressure and the absorbed power. The particle is a silica nanosphere with $R=200 \mathrm{~nm}$, the residual gas is helium at $T_{g}=300 \mathrm{mK}$, the thermal accommodation factor has been set to $\alpha=0.4$, and the emissivity has been set to $\epsilon_{\mathrm{abs}}=0.1$.

tion factor $0<a<1$. The underlying idea is that the scattering of a gas molecule off the nanoparticle is not elastic: the particle is assumed to partially thermalize with the nanoparticle before being reemitted. This model of scattering is abundantly supported by experimental literature [37].

The two baths lead to different mechanical damping rates:

$$
\begin{gathered}
\Gamma_{i}=\frac{4 \pi}{3} \frac{m R^{2} v_{t} P_{g}}{k_{B} T_{i} m_{s}}, \\
\Gamma_{e}=\frac{\pi}{8} \sqrt{\frac{T_{e}}{T_{i}}} \Gamma_{i}
\end{gathered}
$$

where $m_{s}$ is the mass of the particle. The force noise can then be calculated as

$$
S_{f f, \mathrm{~g}}=4 k_{B} m_{s}\left(\Gamma_{i} T_{i}+\Gamma_{e} T_{e}\right) .
$$

There will also be, even in the absence of detection, a force noise due to recoil from emission of blackbody radiation. Following calculations similar to the one leading to Eq. (2), one arrives at the formula [36]

$$
S_{f f, \mathrm{BB}}=\frac{160}{\pi} \frac{R^{3} k_{B}^{6}}{c^{5} \hbar^{4}} \operatorname{Im} \frac{\epsilon_{\mathrm{BB}}-1}{\epsilon_{\mathrm{BB}}+2} T^{6} .
$$

It is easy to check that this contribution is exceedingly small compared to the one of the gas for any realistic set of parameters, except under the condition of extremely low pressure below $10^{-15} \mathrm{mbar}$ and relatively high power. The reason is that photons can remove efficiently energy but at the same they carry very little momentum. An even smaller contribution arises from the scattering and absorption of blackbody radiation coming from the cold environment at temperature $T_{g}$. 


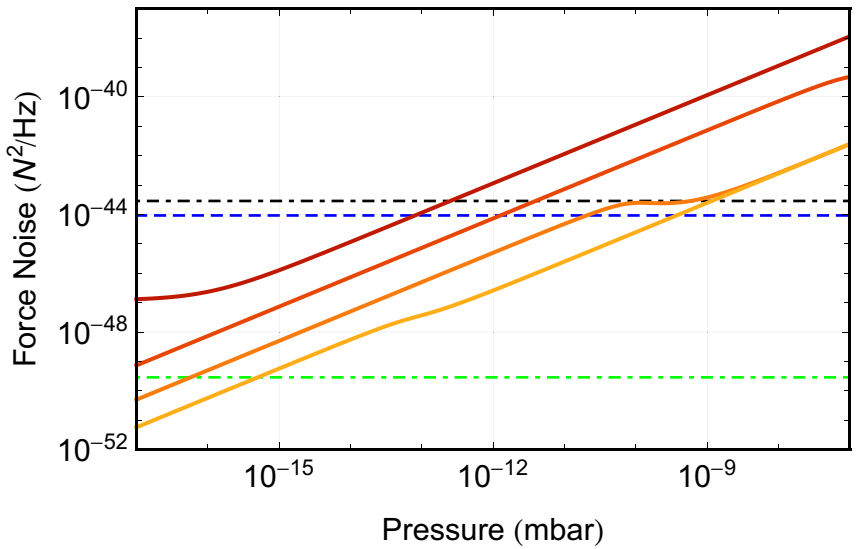

FIG. 3. Thermal force noise as a function of the residual gas pressure. It includes the effects of both gas collisions and blackbody radiation. Thick solid curves refer from top to bottom to an absorbed power of $10^{-10}, 10^{-14}, 10^{-18}$, and $10^{-22} \mathrm{~W}$. Blackbody radiation is always negligible except for the flattening of the $10^{-10} \mathrm{~W}$ curve at low pressure. As in Fig. 2, the particle is a silica nanosphere with $R=200 \mathrm{~nm}$, the residual gas is helium at $T_{g}=300 \mathrm{mK}$, the thermal accommodation factor has been set to $\alpha=0.4$, and the blackbody emissivity has been set to $\epsilon_{\mathrm{abs}}=0.1$. As a reference we also plot the estimated contribution from the Paul trap bias noise (middle horizontal dashed blue line, see text for details), the CSL force noise for $r_{c}=10^{-7} \mathrm{~m}$ and $\lambda=10^{-10} \mathrm{~Hz}$ (top dot-dashed black line), and GRW values $r_{c}=10^{-7} \mathrm{~m}$ and $\lambda=1 \times 10^{-16} \mathrm{~Hz}$ (bottom dot-dashed green line).

Figure 3 shows the thermal force noise as a function of gas pressure for different values of the absorbed power. Clearly, going to sufficiently low pressure will eventually suppress thermal noise below any detectable level. For instance, for the lower value of the pressure reported in literature $10^{-17} \mathrm{mbar}$ [38] and absorbed power $10^{-18} \mathrm{~W}$, the thermal noise would compare to the extremely tiny effect of CSL according to the GRW values. Unfortunately, other effects become dominant in this regime, in particular, electrical noise in the Paul trap and backaction noise from the detection.

\section{Ambient vibrational noise}

In the following we will assume that ambient vibrational noise is negligible. Because of the smallness of the particle considered here, this assumption is rather reasonable. To give a rough idea, let us consider a typical figure for standard seismic noise at level of $S_{a}=10^{-6} \mathrm{~m} / \mathrm{s}^{2} / \sqrt{\mathrm{Hz}}$. For a silica nanosphere with $R=200 \mathrm{~nm}$, the corresponding force noise would be $S_{f}=7 \times 10^{-23} \mathrm{~N} / \sqrt{\mathrm{Hz}}$ at the resonance frequency, roughly equivalent to the CSL noise at $r_{C}=10^{-7} \mathrm{~m}$ and $\lambda=10^{-11} \mathrm{~Hz}$. This suggests that a moderate and feasible mechanical isolation of the order of $60 \mathrm{~dB}$ would be enough to suppress seismic noise well below the CSL noise considered in this paper. However, seismic as well as acoustic noise could influence the experiment in other ways. For instance, the need to lock an optical cavity would impose additional and stricter requirements on the total noise rms at low frequency, but its effect depends drastically on how the cavity is engineered. As such, it will not be taken into account quantitatively in the rest of the paper.

\section{DETECTION SCHEMES}

Here we come to the key issue we wish to study in this paper, namely, how to choose and to optimize the detection of the particle. We will consider three detection options. An optical cavity readout is discussed in Sec. III B, an optical tweezer is considered in Sec. III C, and an electrical readout based on a superconducting quantum interference device (SQUID) is considered in Sec. IIID. Before going into the details of the three techniques, we will analyze in Sec. III A the general features of two different measurement strategies, a stationary continuous one and a stroboscopic reheating, finding that they are in principle equivalent. Based on this conclusion we will focus in the following sections on the continuous measurement strategy.

\section{A. Continuous and stroboscopic measurement}

In this section we will try to compare continuous and stroboscopic measurements. Some relevant considerations can be done regardless of the specific detection technique.

In a steady-state approach the position of the trapped mechanical harmonic oscillator is continuously measured. The acquired signal is fast Fourier transformed (FFT) and periodograms are averaged to provide an estimation of the PSD. An example of application of this method in the context of testing collapse models is given by recent cantilever experiments [20].

In general, there will be two contributions to the PSD, a wide-band position measurement noise $S_{x x}$ and the true oscillator noise with PSD given by $|\chi(\omega)|^{2} S_{f f}$ where

$$
\chi(\omega)=\frac{1}{m_{s}\left[\left(-\omega^{2}+w_{0}^{2}\right)-i \omega \Gamma\right]}
$$

is the Lorentzian mechanical susceptibility. For high- $Q$ systems, and provided $S_{x x}$ is low enough, the oscillator noise will be dominant around the resonant frequency $\omega_{0}$, over a given bandwidth $\Delta f=\Delta \omega / 2 \pi$ which depends on the actual values of $S_{x x}$ and $S_{f f}$. If we define $\Delta f=\left|f_{2}-f_{1}\right|$, where $f_{1,2}=$ $\omega_{1,2} / 2 \pi$ are the frequencies at which $S_{f f}|\chi(\omega)|^{2}=S_{x x}$, we find that

$$
\Delta f=\frac{1}{2 \pi m_{s} \omega_{0}} \sqrt{\frac{S_{f f}}{S_{x x}} .}
$$

The estimation of $S_{f f}$ is inferred from the data available within this bandwidth, for instance, by fitting the estimated PSD. The relative uncertainty on $S_{f f}$ will be of the order of $\approx 1 / \sqrt{t_{m} \Delta f}$ where $t_{m}$ is the measurement time. For instance, for a single FFT with acquisition time $t_{m}$ the frequency resolution is $1 / t_{m}$ and therefore there will be about $n=t_{m} \Delta f$ independent samples for the determination of $S_{f f}$. The same dependence on $t_{m}$ is obtained if FFT averaging is implemented. This simplified argument can be made more rigorous by means of Wiener filter theory [39].

Now, let us assume that the detection is Heisenberg limited, i.e., $S_{f f} S_{x x}=\hbar^{2}$ [40], and that thermal noise is negligible. In this ideal case the force noise is completely determined by the measurement backaction, and therefore by the strength of the measurement. By using the Heisenberg condition to eliminate 
$S_{x x}$ from Eq. (9), we find

$$
\Delta f=\frac{S_{f f}}{2 \pi m_{s} \hbar \omega_{0}} .
$$

We can connect this ideal measurement bandwidth with the phonon heating rate $\gamma$ due to $S_{f f}$, which is generally defined by the relation

$$
\gamma=\frac{S_{f f}}{4 m_{s} \hbar \omega_{0}} .
$$

By comparing Eqs. (10) and (11) we can conclude that $\Delta f=$ $2 \gamma / \pi \simeq \gamma$, i.e., the effective measurement rate $\Delta f$ is set by the phonon heating rate $\gamma$. In the nonideal case other force noise sources are present. We may define a factor $N$ such that $S_{f f} S_{x x}=N^{2} \hbar^{2}$ and performing a similar analysis we conclude that $\Delta f=2 \gamma /(\pi N) \simeq \gamma / N$.

We turn now to the stroboscopic reheating strategy, which is described, for instance, in Refs. [28,41]. Here one works in nonstationary conditions. In a first step the trapped particle is monitored with high sensitivity, and feedback cooling is applied to prepare the system in a state as cold as possible with mean phonon number $\left\langle n_{1}\right\rangle$. Subsequently, feedback is switched off and the oscillator will reheat due to the force noise $S_{f f}$. A possible advantage of this approach is that during the free reheating evolution one is allowed to switch off the detection, therefore avoiding completely measurement backaction. After a given evolution time $t$, the system energy is measured again and the heating rate $\gamma$ is inferred through the relation

$$
\gamma=\frac{\left\langle n_{2}\right\rangle-\left\langle n_{1}\right\rangle}{t}
$$

where $\left\langle n_{2}\right\rangle$ is the mean phonon after the free evolution.

How fast can a measurement of $\gamma$ and hence $S_{f f}$ be? Again, let us start with an ideal Heisenberg limited detector, in the absence of other noise sources. Under these ideal conditions one may in principle perform feedback cooling to the ground state, so that in the initial state $\left\langle n_{1}\right\rangle \simeq 0$ with uncertainty $\sigma_{n_{1}}=1 / 2$. After a free evolution time $t$ the energy is measured again, giving $\left\langle n_{2}\right\rangle=\gamma t$ with uncertainty $\sigma_{n_{2}} \simeq \sqrt{\left\langle n_{2}\right\rangle}$. The relative uncertainty on the estimation of $\gamma$ will be

$$
\frac{\sigma_{\gamma}}{\gamma} \simeq \frac{1}{\sqrt{\left\langle n_{2}\right\rangle}}=\frac{1}{\sqrt{\gamma t}} \text {. }
$$

From a statistical point of view we can thus interpret $\gamma t$ as an effective number of independent samples for the experimental estimation of $\gamma$, so that $\gamma$ can be interpreted as an effective measurement rate. We have thus arrived essentially at the same expression of the continuous measurement case, apart from constants of order 1, meaning that the time required to estimate $S_{f f}$ or equivalently $\gamma$ with a given accuracy is the same. In the case of nonideal detection, we proceed as before by defining a factor $N$, and the initial state and the final state will be affected by a larger uncertainty. As in the continuous case, this leads to a reduction of the measurement rate to $\gamma / N$.

In conclusion, the two strategies appear to be roughly equivalent from a fundamental point of view. The choice of one instead of the other one will depend mostly on technical implementation aspects. Continuous strategies are in principle easier to implement, being based on a stationary

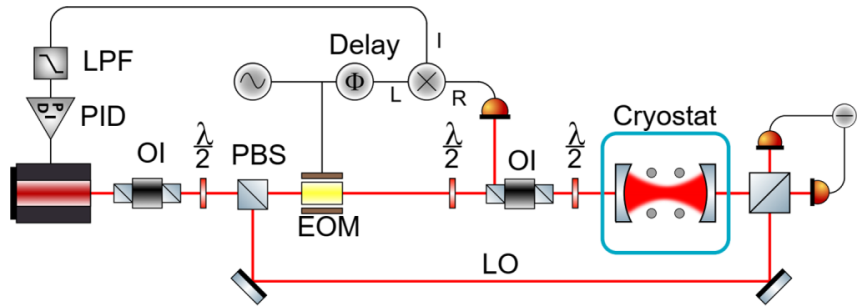

FIG. 4. Simplified scheme of the experiment. A Nd:Yag laser at $1064 \mathrm{~nm}$ is locked on the fundamental mode of an optical cavity by means of a standard Pound-Drever-Hall technique. A silica bead is held at the center of the cavity field by a linear Paul trap. Measurement of the particle dynamic is obtained by homodyning the transmitted cavity field.

state. However, measurements at very low coupling could be challenging for technical reasons and require high stability of the trap frequencies. Stroboscopic measurements do not suffer from the last problem but require the ability to deal with the transients associated to switching detection on and off, which can be extremely challenging in practice.

\section{B. Optical cavity}

Here we consider an optical cavity exploited as a pure displacement sensor. Contrary to the tweezer approach, the cavity is sensitive mainly to one degree of freedom of the particle motion. The requirements to achieve a meaningful measurement in the CSL context are quite stringent and will drive the design of the detection in a direction that is quite different from the typical optomechanical framework.

We show in Fig. 4 a schematic view of the experiment. A nanoparticle is held at the center of an optical cavity by a Paul trap. The transmitted cavity field is then analyzed by homodyne detection. In general, the presence of a dielectric in an optical cavity causes the optical resonance to be downshifted in frequency. For a spherical Rayleigh particle this is simply given by $[36,42] \delta \omega=g_{o} \cos [\phi]^{2}$, where $\phi=k x$ is the position of the particle in the cavity standing wave, with $k$ the wave number, and $g_{o}$ is the characteristic optomechanical coupling strength. Thus, the particle dynamics can be monitored by an optical phase sensitive detection. The highest sensitivity is obtained at $\phi=\pi / 4$ (maximum of $\frac{\partial \delta \omega}{\partial x}$ ) where, to first order, the transduction is linear. At the same time, however, dipole forces provide a trapping potential with a trap frequency at the antinode $(\phi=0)$ given by [43]

$$
\omega_{t}=\frac{2 \hbar k^{2} g_{o}}{m_{s}} n_{c}
$$

Here, $n_{c}$ is the intracavity photon number. This potential is exploited in many applications [44-46]; here, however, it represents an unwanted perturbation that can easily dominate over the Paul trap potential.

This very simple description already allows us to formulate two stringent requirements. First, assuming that the Paul trap and the cavity can be accurately aligned so that $\langle\phi\rangle=\pi / 4$, the rms displacement needs to be small enough to keep the cavity transduction in the linear regime. This displacement has to include both the thermal secular motion and the driven 
micromotion. Second, the optical potential needs to be negligible compared to the Paul trap potential. At the optimal position for the detection the optical potential exerts a force displacing the steady-state position of the particle moving it away from the center of the Paul trap potential. As a consequence we need $\omega_{t}<\omega_{0}$. This requirement strongly limits the maximum intracavity power allowed, pointing to a low finesse cavity as the best choice.

Another critical aspect is the backaction introduced by the detection which will ultimately limit the force noise sensitivity. The main sources of backaction are radiation pressure shot noise and recoil heating [47]; however, cavity dynamical effects must be accounted for since a nonvanishing detuning will introduce optical spring and damping.

In the following we are going to show the expected sensitivity assuming an ideal homodyne detection, which is the best case scenario due to technical aspects. Laser frequency noise represents a major technical limitation. For a resonant optical drive, it can be considered as an additive noise limiting the sensitivity far above the shot noise [48]. In order to leave unaltered the particle signal in the homodyne, the cavity lock bandwidth has to be much smaller than $f_{0}$. This imposes an extremely demanding requirement in terms of displacement noise of the cavity mirrors, especially considering the cryogenic environment.

We consider an asymmetric low finesse $(\approx 1000)$ cavity undercoupled on the injection side. The cavity length is $L=$ $15 \mathrm{~mm}$, has a waist of $w_{s t}=62 \mu \mathrm{m}$, and is coupled to a 200-nm radius silica nanoparticle. Assuming a 1064-nm laser driving the cavity, we have a single-photon optomechanical coupling $g / 2 \pi=x_{z p f} \mathrm{~kg}_{o} / 2 \pi \simeq 5 \mathrm{~Hz}$. We consider optical input powers ranging from 0.1 to $20 \mu \mathrm{W}$ for which we can estimate the power absorbed by the particle. Assuming a pressure of $10^{-13} \mathrm{mbar}$, which should be achievable in an ultracryogenic environment [49], the particle equilibrium temperature is expected to be between 20 and $70 \mathrm{~K}$ following a $W_{\text {abs }}^{1 / 5}$ power law since it is completely determined by blackbody radiation, as shown in Sec. II D.

We show in Fig. 5 the expected PSD of the homodyne detection of the transmitted beam normalized to the shot noise for an input power of $10 \mu \mathrm{W}$ along with all main contributions. Despite an effective bath temperature of $\approx 20 \mathrm{~K}$, the total PSD is dominated by photon recoil. This remains true for all input powers considered. Also shown in Fig. 5 is the estimated limit to sensitivity due to laser frequency noise. Typical spectra for high stability Nd:Yag lasers give $S_{v v}(f) \simeq$ $4 \times 10^{8} / f^{2} \mathrm{~Hz}^{2} / \mathrm{Hz}$.

In order to compare the sensitivity to CSL, we calculate the total force noise acting on the particle and express it in terms of collapse rate $\lambda$ assuming a characteristic length $r_{C}=$ $10^{-7} \mathrm{~m}$. Therefore, the collapse rate that will give a signal to noise ratio of 1 is summarized in Fig. 6, where we plot the imprecision noise as a function of the collapse rate for both shot-noise limited and frequency noise limited detection. For the former case, imprecision noise decreases for an increasing power as is typical for a shot-noise limited detection. For the latter case, the imprecision noise is relatively constant. However, the CSL noise sensitivity is always reduced for an increasing power since the system is dominated by photon recoil.

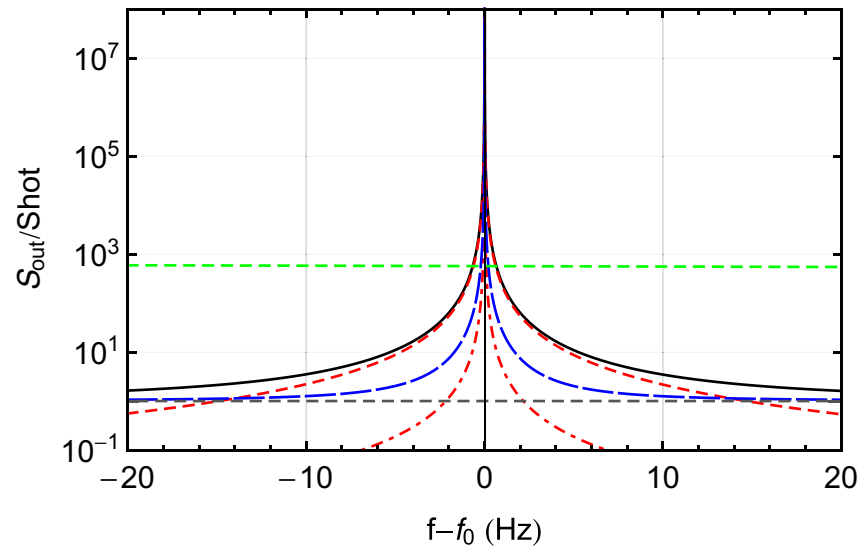

FIG. 5. Cavity output homodyne spectrum normalized to shot noise. Black is the total noise, blue (long-dashed) is the quantum noise, red dot-dashed is the thermal noise, and red dashed is the photon recoil. Finally, the (dashed) horizontal green line represents the typical frequency noise that could hinder the particle detection.

Another important parameter is the measurement bandwidth since it directly impacts measurement time and as a consequence the requirements on stability on all other parameters. This is shown in Fig. 6 as well. For a frequency noise limited detection this turns out to be $\approx 150 \mathrm{mHz}$ for the smaller power and up to $\approx 2 \mathrm{~Hz}$ for the highest. For shot-noise limited readout the situation would be much more favorable with a bandwidth varying from $\approx 400 \mathrm{mHz}$ to $\approx 60 \mathrm{~Hz}$. Of course, achieving this seems rather challenging especially considering the cryogenic environment. Indeed, for the configuration considered, the shot-noise level would correspond to a relative displacement noise of the cavity mirrors of the order of $\approx 10^{-33} \mathrm{~m}^{2} / \mathrm{Hz}$.

So far we have assumed a rather optimal scenario; here we discuss some criticalities. First, as stated before, the rms displacement of the particle needs to be sufficiently small to remain in the linear transduction region of the cavity, that is, $\Delta x=\left(k_{B} T_{\text {eff }} / m \omega_{0}^{2}\right)^{1 / 2} \leqslant \lambda / 16$. For the parameters

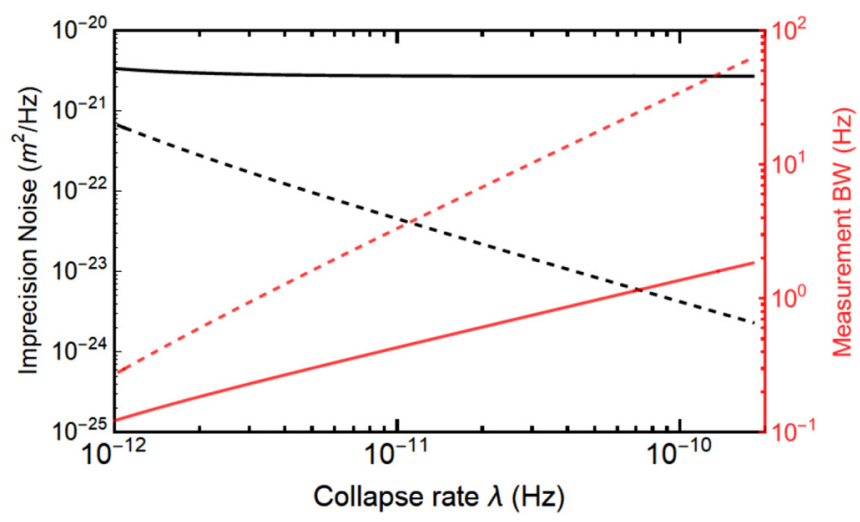

FIG. 6. Imprecision noise and measurement bandwidth as a function of the collapse rate $\lambda$ that would provide a signal to noise ratio of 1 given the estimated total force noise acting on the particle and assuming $r_{c}=10^{-7} \mathrm{~m}$. Continuous lines refer to a frequency noise limited detection while dashed lines refer to a shot-noise limited one. See main text for more details. 
TABLE I. Main parameters considered for the cavity readout.

\begin{tabular}{ll}
\hline \hline Parameter & \multicolumn{1}{c}{ Value } \\
\hline$\lambda$ & $1064 \mathrm{~nm}$ \\
$L$ & $15 \mathrm{~mm}$ \\
$w_{s t}$ & $62 \mu \mathrm{m}$ \\
Finesse & 1000 \\
$P_{\text {in }}$ & $0.1-20 \mu \mathrm{W}$ \\
\hline \hline
\end{tabular}

considered here we have $\Delta x \simeq 0.4 \lambda$ meaning that additional active feedback is required in order to reduce the particle fluctuations. To meet this requirement the mechanical quality factor needs to be reduced by a factor 100. This seems reasonable considering that its value is expected to be $\approx 10^{9}$ at $P_{g}=10^{-10} \mathrm{mbar}$ and increases inversely proportional to pressure.

The optical spring near resonance has a linear dependence on detuning; we can impose a shift in frequency equal to the mechanical linewidth $\Gamma_{\text {tot }}$ from which we can get a limit on the detuning for given cavity parameters, that is,

$$
\Delta \leqslant \frac{c}{2 P_{\text {in }}} \frac{m_{s} w_{0} \kappa^{4}}{2 g_{o}^{2} k \kappa_{\text {in }}} \Gamma_{\text {tot }},
$$

where $k$ is the wave number, $\kappa$ is the cavity half linewidth, and $\kappa_{\text {in }}$ is the contribution to it due to the input port. For our parameters and an input power of $10 \mu \mathrm{W}$ this is roughly $\Delta \leqslant 10^{-7} \kappa \simeq 0.4 \mathrm{~Hz}$, which is an extremely demanding requirement. Just to give a reference the maximum shift is $\approx 1 \mathrm{~Hz}$. Since we are in the deeply bad cavity regime the same kind of limit imposed using the optical damping is much less stringent. The situation becomes more relaxed if we assume some kind of active feedback increasing the mechanical linewidth. However, this increase needs to be by a factor $\approx 1000$ at least. At the same time, any blue detuning will give rise to dynamical instability. Thus a small but finite detuning seems rather necessary.

Some final considerations are required concerning the optical power considered. The plots in Fig. 6 summarizing the performance in terms of sensitivity to CSL noise consider input powers ranging from 0.1 to $20 \mu \mathrm{W}$. The upper bound to the input power is due to the optical potential as previously discussed. The lower bound is somewhat more free but will ultimately be set by technical considerations concerning cavity locking. Indeed, a simple locking scheme as depicted in Fig. 4 is quite challenging to implement with the minimum power considered. However, more complicated locking schemes could be implemented. For example, the cavity could be locked to a higher-order cavity mode (i.e., a $\mathrm{TEM}_{01}$ ) which has a node along the cavity axis and is thus not coupled to the particle to first order, while a second laser is offset locked to be resonant to the cavity fundamental mode. A detailed study of the optimal optical setup is not the main focus of this paper and will be the topic of future research.

As a conclusion to this section we list in Table I the important parameters and we summarize here some key aspects and requirements necessary to implement the cavity detection of the nanoparticle motion.
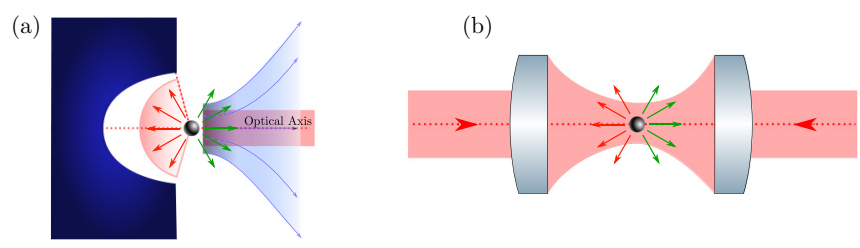

FIG. 7. (a) Detection using a paraboloidal mirror and (b) detection using a dual counterpropagating beam setup.

(1) The cavity and Paul trap need to be accurately aligned so that $\langle\phi\rangle=\pi / 4$.

(2) The intracavity power has an upper bound due to the gradient force trapping potential, i.e., $\omega_{t}<\omega_{0}$.

(3) The particle thermal motion needs to be reduced to guarantee $\Delta x_{\mathrm{th}} \leqslant \lambda / 16$.

(4) The accuracy and stability of the detuning of the optical field need to be $\Delta \leqslant 10^{-7} \kappa$.

(5) The cavity stability and displacement noise have to be controlled to allow a lock bandwidth much less than $\omega_{0}$.

\section{Optical tweezer}

We consider two different tweezer-based detection setups depicted in Fig. 7. Specifically, we consider a nanoparticle in a Paul trap with trap frequency $\omega_{0}$ which is monitored either continuously or stroboscopically using a tweezer. The trapped nanoparticle scatters light which is collected using optical elements and then directed towards the detector; similarly as in the cavity case we again consider the situation where the scattered light interferes with a local oscillator in a homodyne scheme [50]. For concreteness, we consider a laser wavelength $\lambda=1550 \mathrm{~nm}$ and assume a spherical silica $\left(\mathrm{SiO}_{2}\right)$ nanoparticle of radius $R=200 \mathrm{~nm}$.

The Paul trap potential, $U_{\text {Paul }}$, can be significantly perturbed by the optical potential, $U_{\text {optical }}$, as well as by the effective potential $U_{\text {scatt }} \propto F_{\text {scatt }} x$ generated by the nonconservative scattering force $F_{\text {scatt }}$ oriented in the propagation direction $x$ of the laser beam [51]. The presence of the optical potential $U_{\text {opt }} \propto \omega_{\text {optical }}^{2} \propto P$ results in a change of the trap frequency, i.e., $\omega_{0}^{2}+\omega_{\mathrm{opt}}^{2}$, where $\omega_{\mathrm{opt}}$ and $P$ denote the optical frequency and laser power, respectively. On the other hand, the effective scattering potential $U_{\text {scatt }}$ displaces the origin of the combined Paul and optical trap; if the displacement is too large the nanoparticle can leave the harmonic region of the trap, possibly even resulting in particle loss. For the considered nanoparticle of size $R=200 \mathrm{~nm}$, noting that $U_{\text {optical }} \propto R^{3}$ and $U_{\text {scatt }} \propto R^{6}$, we find the strongest constraint coming from the requirement $\left|\partial_{x} U_{\text {scatt }}\right| \lesssim\left|\partial_{x} U_{\text {Paul }}\right|$; specifically, assuming a Paul trap frequency $\omega_{0}=2 \pi \times 1 \mathrm{kHz}$ we are limited to powers of $P \lesssim 1 \mathrm{nW}$. To avoid the problem related to the scattering potential $U_{\text {scatt }}$ one can, however, consider two counterpropagating beams which could be implemented using the lens setup shown in Fig. 7(b); requiring $U_{\text {optical }}<U_{\text {Paul }}$ we then find a much less restrictive condition on the laser power, i.e., $P \lesssim 1 \mathrm{~mW}$.

Ideally we would like to set the laser power $P$ to the value which minimizes the sum of the backaction and imprecision noise [40]. However, the optimal value of $P$ is relatively low and sources of dark noise present in physical detectors must 


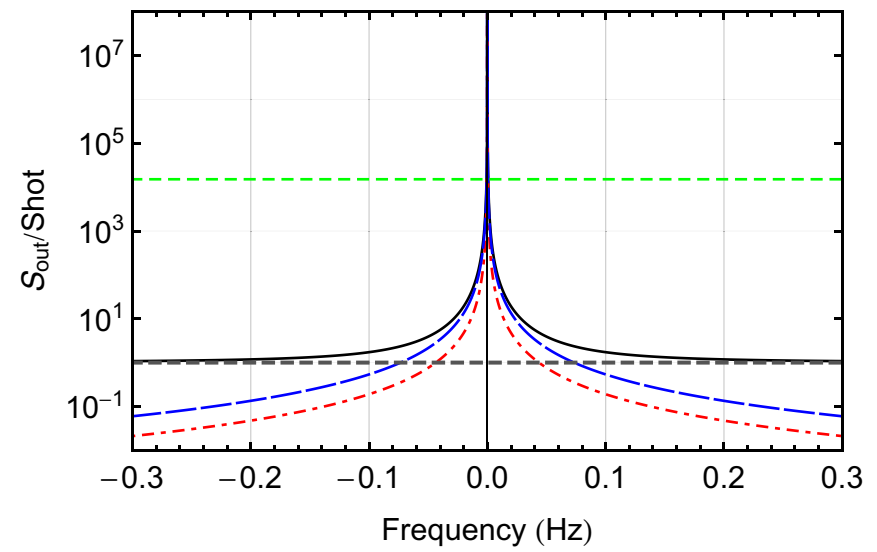

FIG. 8. Optical tweezer homodyne spectrum normalized to shot noise. Black is the total noise, blue (long-dashed) is the quantum noise (photon recoil and backaction), and red (dot-dashed) is the thermal noise. Finally, the (dashed) green horizontal line represents the noise floor estimated using NEP to quantify the detector's dark noise which is expected to significantly hinder the detection.

be taken into account. From the laser power $P$ and the particle position $x$ one can then estimate the photocurrent impinging on the detector; we quantify it by the total efficiency $\eta$, and denote the detected power by $P_{\text {det }}$. In particular, adopting a simple semiclassical calculation we can find the conversion factor between the detected power and the particle position [50]. We compare $P_{\text {det }}$ to the detector's dark noise, which can be estimated by considering the noise equivalent power (NEP) of the detector [52]. The NEP noise floor, although not an intrinsic limitation of the tweezer setup, is expected to be the dominant contribution to the noise floor (see Fig. 8).

The fundamental noise floor in a tweezer setup, similarly as in the cavity setup, is given by the shot noise. The nanoparticle motion is affected by noise from gas collisions, photon recoil, and backaction. In Fig. 8, we show the expected homodyne spectrum for the tweezer case. We are assuming a pressure of $10^{-13}$ mbar, a numerical aperture NA $=0.6$ limited by reasonable trap geometries, and an input power of $500 \mathrm{nW}$ at a wavelength of $1550 \mathrm{~nm}$. With this power we estimate a particle equilibrium temperature of $T \simeq 60 \mathrm{~K}$, quite similar to the cavity case shown in Fig. 5. Already at such low power the particle dynamics is dominated by quantum noise.

To summarize the performance and allow an easy comparison, we show, in Fig. 9, the imprecision noise and measurement bandwidth as a function of detectable $\lambda$, in analogy to Fig. 6. The main advantage of the tweezer lies in the much wider range in acceptable power. Contrary to the cavity case, the maximum sensitivity is obtained in the position of maximum intensity of the optical field. This allows one to fully exploit the canceling forces due to the counterpropagating beams. However, the resulting displacement sensitivity is lower with a direct impact to the attainable measurement bandwidth.

The most critical aspect of the tweezer approach lies in the requirements on the positioning accuracy of the two objective lenses. In order for the suppression of the scattering force to be effective, the position and size (i.e., nonidealities) of the waist of the two lenses needs to be close to a fraction of

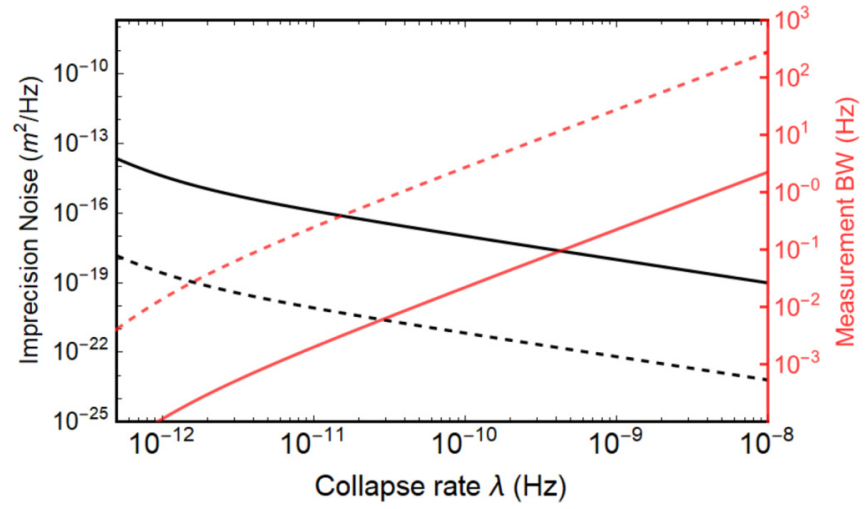

FIG. 9. Imprecision noise and measurement bandwidth as a function of the collapse rate that would provide a signal to noise ratio of 1 given the estimated total force noise acting on the particle and assuming $r_{C}=10^{-7} \mathrm{~m}$. Continuous lines refer to a NEP noise limited detection while dashed lines refer to a shot-noise limited one.

the nominal waist, which is of the order of $\approx 2 \mu \mathrm{m}$ (i.e., half the radius of the core of a telecom single mode fiber). While this is standard practice at room temperature, maintaining the alignment in an ultracryogenic environment is a completely different endeavor.

As for the previous section we list in Table II important parameters and summarize the key aspects and requirements of the tweezer approach.

(1) The tweezer and Paul trap need to be aligned with an accuracy better than the optical waist.

(2) The counterpropagating configuration is required.

(3) Any misalignment of the two beams will result in a dramatic increase of the scattering force.

\section{Electrical readout with a SQUID}

Instead of detecting the oscillating particle using an optomechanical setup, it has been proposed to use a direct electrical detection [53]. To this end, a pair of electrodes, for instance, two endcaps, are used to detect the motion of the particle along the axis orthogonal to them. The electrical signal induced in the electrodes could be eventually read out by a SQUID current sensor. SQUIDs are the best electrical amplifiers available in a wide range of frequencies, and are a natural choice when working at low temperature and low frequency.

Unfortunately the electromechanical coupling of the above scheme is very weak for a current-based detection at low frequency. As shown in Refs. [53,54], the motion $x$ of the

TABLE II. Main parameters considered for the tweezer readout.

\begin{tabular}{ll}
\hline \hline Parameter & \multicolumn{1}{c}{ Value } \\
\hline$\lambda$ & $1550 \mathrm{~nm}$ \\
$\mathrm{NA}$ & 0.6 \\
$w_{s t}$ & $1.9 \mu \mathrm{m}$ \\
$\eta$ & 0.0003 \\
$\mathrm{NEP}$ & $1.55 \mathrm{pW} / \sqrt{\mathrm{Hz}}$ \\
$P_{\text {in }}$ & $1 \mathrm{nW}$ to $1 \mathrm{~mW}$ \\
\hline \hline
\end{tabular}




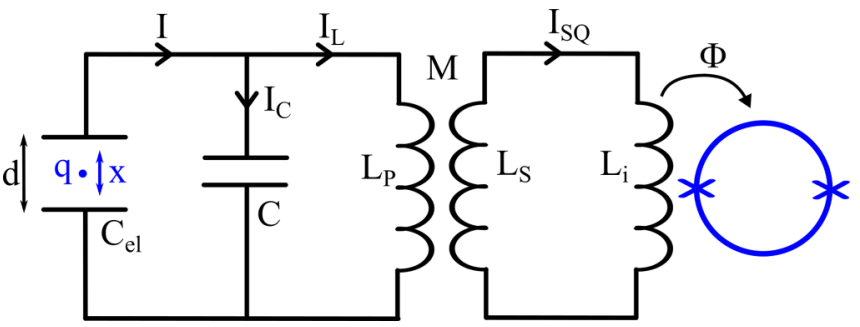

FIG. 10. Scheme of a SQUID-based detection of the levitated particle. The motion of the charge $q$ induces a current $I$ in the electrodes of capacitance $C_{\mathrm{el}}$. The current is enhanced by a low-loss superconducting transformer with mutual inductance $M$ and high inductance ratio $\left(L_{p} \gg L_{s}\right)$ before being measured by a SQUID of input inductance $L_{i}\left(L_{i} \simeq L_{s}\right)$. A further way to boost the current and improve impedance matching is to add a capacitor $C\left(C \gg C_{\mathrm{el}}\right)$ in order to tune the $L C$ resonance to the mechanical resonance of the trapped particle.

particle with charge $q$ will induce a current in the circuit connected to the electrodes:

$$
I=\frac{q}{d} \dot{x}=\frac{\omega q}{d} x,
$$

where $d$ the effective gap between the electrodes and $\omega$ is the oscillation frequency. Furthermore, it was shown that the mechanical oscillator, as seen from the electrical circuit, is dynamically equivalent to a $R L C$ oscillator with effective inductance $L_{m}=m_{s} / \beta^{2}$ and capacitance $C_{m}=1 / \omega_{0}^{2} L_{m}$, where $\beta=q / d$.

For a reasonable set of parameters envisaged for the experiment, $m_{s}=6 \times 10^{-17} \mathrm{~kg}$ (standard 200-nm-radius $\mathrm{SiO}_{2}$ particle), $q=10^{3} e, d=300 \mu \mathrm{m}$, and $\omega_{0} / 2 \pi=1 \mathrm{kHz}$, one obtains effective $L C$ inductance and capacitance $L_{m}=$ $2 \times 10^{8} \mathrm{H}$ and $C_{m}=10^{-16} \mathrm{~F}$. For comparison a typical SQUID sensor has an input inductance $L_{i} \simeq 10^{-6} \mathrm{H}$, which means a huge impedance mismatch by 14 orders of magnitude. In fact, with a typical input current noise $S_{i} \simeq 0.1 \mathrm{pA} / \sqrt{\mathrm{Hz}}$, a direct coupling of the electrodes to a SQUID would lead to a very poor displacement resolution $S_{x} \simeq 30 \mu \mathrm{m} / \sqrt{\mathrm{Hz}}$.

One of the reasons for such a significant mismatch is that, according to Eq. (16), the displacement to the current transfer function is proportional to $\omega$, which makes this scheme very suboptimal at low frequency. This situation is analogous to Faraday detection of magnetic fields.

To overcome this problem, one can explore different strategies. The first is to reduce impedance mismatch, and the second is to resort to other types of amplifier, such as standard field-effect transistor (FET) transimpedance amplifiers or single-electron transistors.

\section{SQUID with untuned transformer}

The simplest way to reduce impedance mismatch to a SQUID is to interpose a superconducting transformer between the electrodes and the SQUID, as shown in Fig. 10. This increases the effective input inductance to a value of the order of the inductance $L_{p}$ of the primary coil. Low-loss superconducting transformers with inductance up to $10 \mathrm{H}, Q \simeq 10^{6}$, and a volume of around $1 \mathrm{~L}$ have been used to match a SQUID to the capacitive transducer of resonant bar gravitational wave

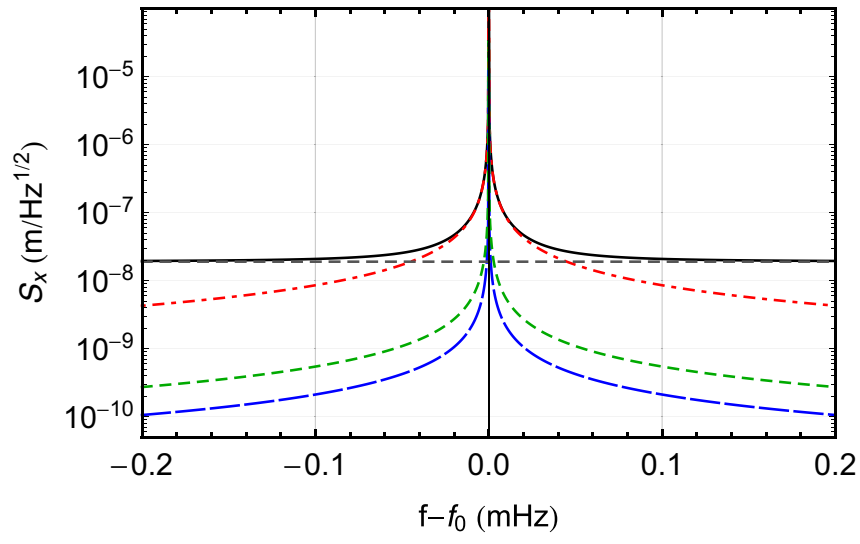

FIG. 11. Spectrum of a levitated particle measured by a SQUID through a superconducting transformer. The particle is a silica nanosphere with $R=200 \mathrm{~nm}$, charge $q=10^{3} e$, the resonance frequency $f_{0}=1 \mathrm{kHz}$, the residual gas is helium at $T_{g}=300 \mathrm{mK}$, and $P_{g}=10^{-12}$ mbar. The SQUID noise is $N_{\hbar}=10$ and the transformer has $L_{p}=10 \mathrm{H}$, coupling $k=0.8$, and $Q=1 \times 10^{6}$. The various noise components are total noise (solid black), gas collision noise (dot-dashed red), electrical noise (dashed green), SQUID backaction (blue long-dashed line), and SQUID imprecision (dashed horizontal gray line). The total force noise around resonance corresponds to a CSL collapse rate $\lambda \simeq 6 \times 10^{-14} \mathrm{~Hz}$.

detectors [55]. Larger values are in principle achievable but are hardly compatible with standard cryostats.

Let us consider as a maximum realistic choice $L_{p}=$ $10 \mathrm{H}$, and assume the transformer geometrical coupling $k=$ $M^{2} /\left(L_{p} L_{s}\right)$ to be close to the maximum allowed value of 1 . For optimal matching the secondary coil inductance should be $L_{s} \simeq L_{i}$, where $L_{i}$ is the SQUID input inductance.

The SQUID can be modeled as a current amplifier with an imprecision current noise, with spectral density $S_{I I}$, and a conjugate backaction voltage noise with spectral density $S_{V V}$. Neglecting cross correlations, a rough approximation for the spectral densities is $S_{I I} \simeq 2 \hbar / L_{i} N_{\hbar}$ and $S_{V V} \simeq 2 \hbar \omega^{2} L_{i} N_{\hbar}$. This way a single noise parameter $N_{\hbar}$ is singled out. The best available SQUIDs have shown effective noise $N_{\hbar} \simeq 10$ which is not far from the Heisenberg limit $N_{\hbar}=1$.

Besides SQUID noise, one has to consider the Nyquist electrical thermal noise of the transformer. At $\mathrm{kHz}$ frequencies the best reported electrical quality factor is $Q \simeq 10^{6}$ [56]. This implies a voltage noise referred to the primary coil, $S_{V V \text { el }}=$ $4 k_{B} T_{g} \omega_{0} L_{p} / Q$. Taking into account the transformer coupling, Nyquist noise is equivalent to a SQUID backaction noise with equivalent noise number $N_{\hbar}^{\prime} \simeq 2 k_{B} T_{g} /\left(\hbar \omega_{0} Q\right)$. It happens that for $T_{g}=300 \mathrm{mK}$ and $Q \simeq 10^{6}$ electrical noise is slightly larger than SQUID backaction noise at $1 \mathrm{kHz}$. Thermal noise is dominant for $f_{0}<1 \mathrm{kHz}$, and becomes negligible for $f_{0} \gg 1 \mathrm{kHz}$.

Having defined the parameters, we can now simulate the spectrum of a levitated nanoparticle coupled to a SQUID through a transformer. Figure 11 shows a spectrum based on the representative set of parameters discussed above. The spectrum shows that the coupling would be so low that both Nyquist noise and backaction noise are much smaller than the gas collision noise, even at a pressure $P_{g}=10^{-12}$ mbar. 


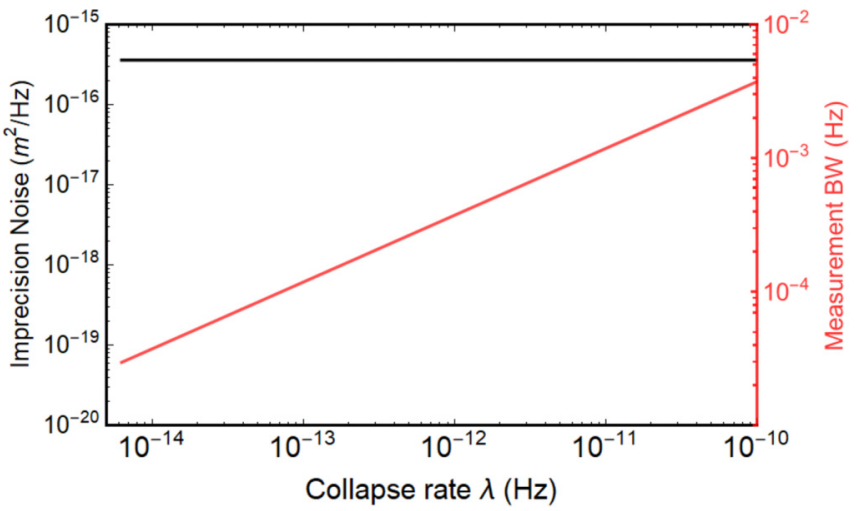

FIG. 12. Displacement noise and measurement bandwidth, expressed as a function of the minimum measurable collapse rate $\lambda$, evaluated at the standard value $r_{C}=10^{-7} \mathrm{~m}$, for the untuned SQUID readout. The parameters are the same as in Fig. 11, except for the gas pressure, which is variable, in order to vary the force noise and thus the detectable $\lambda$.

Assuming the particle to be thermalized, as there is no direct dissipation of energy, the force noise on resonance would allow one to test the CSL model down to $\lambda<10^{-13} \mathrm{~Hz}$. The drawback is that because of low coupling the bandwidth would be extremely narrow, $\Delta f<1 \mathrm{mHz}$.

For the sake of comparison with the optical cavity and tweezer approaches, we construct a bandwidth vs detectable $\lambda$ in analogy to Fig. 6 . In contrast with the optical case, the backaction noise here is negligible and the position noise is fixed by the circuital parameters, so that there is no way to tune the measurement coupling (as changing power in the optical case). We vary the thermal force noise by varying the gas pressure $P$, and calculate the corresponding minimum detectable $\lambda$ and bandwidth. In this way we obtain the plot in Fig. 12.

Clearly, the striking feature of a SQUID-based detection of a nanoparticle in a Paul trap is the extremely narrow bandwidth. In principle such an experiment would not be unfeasible, as it would be possible to acquire tens of independent points in one day. However, this would require the trap frequency to be extremely stable within better than $1 \mathrm{mHz}$ over such a time scale. This is not trivial for an actively controlled trap. In addition, note that the experimental parameters are already quite tight. Lower charge, higher electrode distance, or lower primary coil inductance will all have the effect of further reducing the bandwidth.

Despite the very narrow bandwidth, a SQUID-based detection has the very attractive feature of being effective with any type, material, or size of the charged particle. It would then be possible to levitate much heavier particles, for instance, made of gold or other heavy elements such as platinum or osmium, which would strongly increase the coupling to CSL. For instance, with an osmium particle with $R=400 \mathrm{~nm}$ and the same other parameters of Fig. 11, one would reach a force noise of $\lambda \simeq 3 \times 10^{-16} \mathrm{~Hz}$, close to the GRW limit. Unfortunately, in this case the measurement bandwidth would reach the extremely small value of $\Delta f \simeq 2 \mu \mathrm{Hz}$.

It is fair to say that, besides the very long measurement time, we have completely ignored here the voltage noise in
TABLE III. Main parameters considered for the SQUID readout with untuned superconducting transformer.

\begin{tabular}{ll}
\hline \hline Parameter & Value \\
\hline$q$ & $30 e$ \\
$Q$ & $10^{6}$ \\
$L_{p}$ & $10 \mathrm{H}$ \\
$N_{\hbar}$ & $10 \hbar$ \\
\hline \hline
\end{tabular}

the trap bias line. This is actually expected to become a big issue especially for large charge. To make this contribution negligible in the configurations discussed above, it would be necessary to suppress voltage noise in the bias line to $S_{V}<10 \mathrm{pV} / \sqrt{\mathrm{Hz}}$. Furthermore, we have to consider another nontrivial technical issue. The SQUID electronics must be able to handle the signal due to crosstalk from the ac bias line, which will likely be huge. While this crosstalk can be in principle suppressed by proper cancellation schemes, this may be not so easy to do in practice.

As for the optical detection methods, we list in Table III important parameters and summarize the key aspects and requirements of the SQUID readout.

(1) There is no direct heating from the SQUID, thus the internal temperature can be very close to the bath temperature.

(2) The coupling and measurement bandwidth $(<1 \mathrm{mHz})$ are very low, setting very stringent requirements on the trap stability.

(3) The coupling depends on the particle charge, which needs to be as large as possible.

(4) A massive superconducting inductor, with high quality factor, is required.

(5) The Paul trap bias couples a direct signal into the SQUID, which likely needs to be actively suppressed.

\section{SQUID with a transformer and LC}

To further improve the impedance matching, one can tune a $L C$ resonance to the resonance of the trapped particle. Note that a similar strategy at comparable frequency has been pursued in the readout of resonant bar gravitational wave detectors [55]. Besides the increased technical difficulty, it has been shown that this tuned $L C$ system is really advantageous in terms of bandwidth only if the electrical quality factor is comparable or better than the mechanical quality factor. While this condition was met in the case of Ref. [55], it is definitely not valid in our case, as the largest electrical quality factors at $\mathrm{kHz}$ frequencies are of the order of $10^{6}$, while the mechanical quality factor in the configurations here considered is expected to be of the order of $10^{11}$.

To assess whether this intuitive argument is correct we have performed simulations of a circuit with the same parameters as in the transformer setup, but now the capacitor $C$ shown in Fig. 10 in parallel to the primary inductor is used to tune the $L C$ frequency of the circuit to the mechanical frequency.

The simulation is performed by explicitly writing the Kirchoff equations and the coupled mechanical equation, using as independent variable the mechanical displacement $x$, the currents across capacitor and inductor $I_{C}$ and $I_{L}$, and the current in the SQUID $I_{\mathrm{SQ}}$ and including all relevant noise 


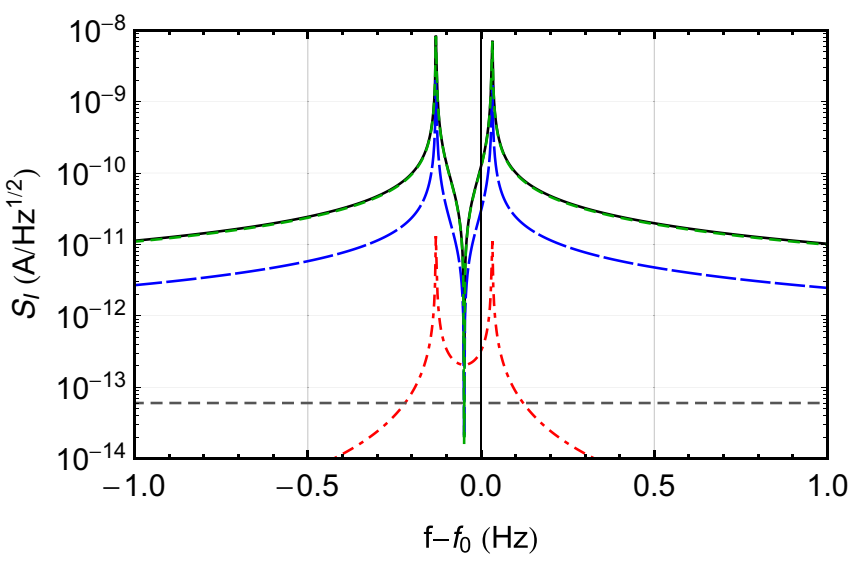

FIG. 13. Spectrum of a levitated particle measured by a SQUID through a superconducting transformer with tuned $L C$ resonance. Parameters are exactly the same as in Fig. 11, except that a capacitor $C$ is added in parallel to the primary inductor to tune the electrical and mechanical frequencies. The spectrum shown here is expressed as current at the input of the SQUID. The color and line type coding for the different contributions is the same as in Fig. 11. Note that the electrical noise (dashed green) is hardly visible as it almost coincides with the total noise (solid black) everywhere except in the narrow antiresonance between the two peaks.

terms. The total output noise is then divided by the force to the SQUID current transfer function to compute the effective total force noise.

First, we have checked that for large detuning between electrical and mechanical frequency we recover the results of the untuned transformer circuit. Then, we have tuned the two frequencies and obtained the interesting behavior shown in Figs. 13 and 14. The two modes hybridize, forming two coupled normal modes. The splitting depends on the electromechanical coupling, but even for large charge $q=10^{3} e$ it is smaller than $1 \mathrm{~Hz}$. In this situation, energy flows back and forth between electrical and mechanical resonators at a rate given by the frequency splitting. However, as the electrical quality factor (here $1 \times 10^{6}$ ) is much larger than the mechanical one (here $Q \simeq 3 \times 10^{11}$ for helium gas at $P_{g}=10^{-12}$ mbar), the noise power is dominated by the

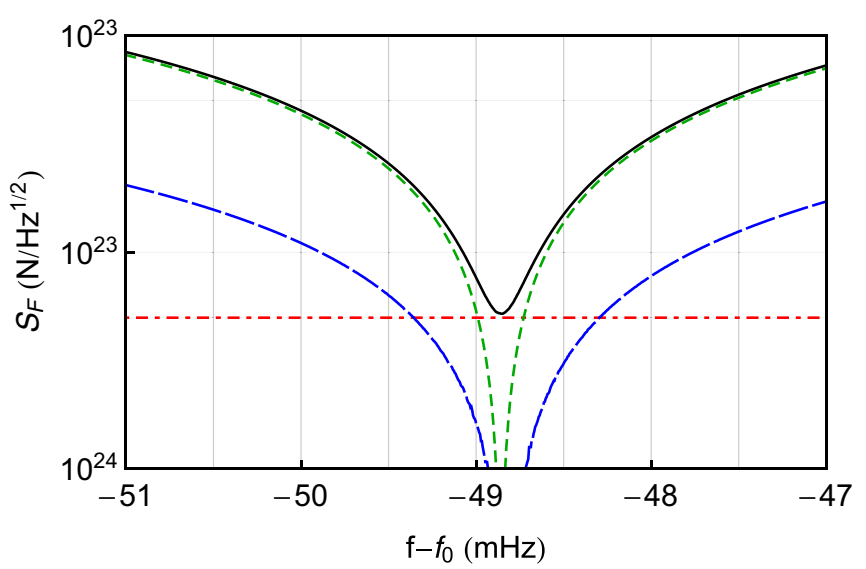

FIG. 14. Spectrum in the same situation as Fig. 13, but now expressed as force noise on the levitated particle and zoomed in a narrow range around the deep antiresonance visible in Fig. 13. former, largely degrading the force noise over almost the whole bandwidth.

Remarkably, there is a very narrow region between the two peaks where the electrical noise features a narrow antiresonance. This point corresponds to the uncoupled mechanical resonant frequency. The antiresonance region is zoomed in Fig. 14, where the spectrum is expressed as force noise on the levitated particle. In a very narrow bandwidth of around $0.2 \mathrm{mHz}$ the electrical noise drops below the very small gascollision mechanical noise.

Within this bandwidth the force noise spectral density is again dominated by the gas, so it is the same as in the untuned case of Fig. 11. Remarkably, the effective bandwidth, despite the very different configuration, is substantially the same. This means that in terms of measurement speed the tuned $L C$ shows no substantial advantage with respect to the untuned case. The situation would slightly improve if the electrical noise were improved by, say, two orders of magnitude. In this case the bandwidth would be limited by SQUID backaction noise.

Finally, note that, similarly to the untuned case (see Fig. 12), the bandwidth would be increased if the pressure were increased to values higher than $10^{-12}$ mbar. This would imply larger force noise, i.e., worse detectable $\lambda$.

\section{Other types of amplifier}

One may also consider using ultralow noise semiconductor amplifiers instead of SQUIDs. Transimpedance FET amplifiers feature an input current noise that can be as low as $\mathrm{fA} / \sqrt{\mathrm{Hz}}$ levels, thus even better than conventional SQUIDs. Unfortunately, in our experiment we need to consider the backaction noise as well. For a good transimpedance FET amplifier it is usually on the order of $1 \mathrm{nV} / \sqrt{\mathrm{Hz}}$. This is more than six orders of magnitude higher than a SQUID at $1 \mathrm{kHz}$. In fact, if we define the "noise number" of a FET amplifier in analogy to a SQUID we find $N_{\hbar} \simeq 10^{6}$. This clearly makes this solution not viable.

A better case is constituted by single electron transistors. These are intrinsic charge sensors, in contrast with SQUIDs that are current sensors, so that the transfer function in a Paul trap setup (displacement to charge) would be flat. In particular, superconducting single-electron transistors (SSETs) can be considered as a dual charge-based version of SQUIDs. They have demonstrated near quantum limited performance, with charge noise down to $10^{-6} e / \sqrt{\mathrm{Hz}}$ over an input impedance of the order of $1 \mathrm{fF}$, which would nicely match the apparent mechanical impedance of the trapped particle. Theoretically, this seems to be the optimal solution for the Paul trap readout.

Unfortunately, the development of SSETs has been much more limited with respect to SQUIDs. SSETs are not available at commercial or semicommercial level, and have been so far implemented only in a very limited range of applications. In general they are much less robust and more sensitive to spurious effects, and it is questionable if there is any chance to operate a SSET in an application requiring huge dynamic range, such as in the readout of a trapped particle.

\section{DISCUSSION}

In this section we will compare the different approaches quantitatively. We have to remark that all three experimental 


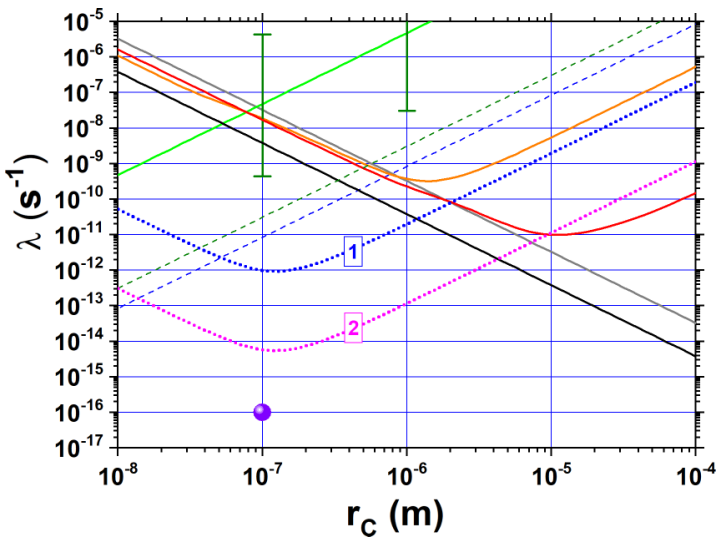

FIG. 15. CSL exclusion plot, where we have reproduced the current bounds already discussed in Fig. 1, together with two representative hypothetical bounds from a levitated nanoparticle. The curve labeled by 1 represents the best scenario considered in this paper based on an optical readout, corresponding to a recoil-limited force noise of $4 \times 10^{-46} \mathrm{~N}^{2} / \mathrm{Hz}$ and a bandwidth of the order of $0.5(0.1) \mathrm{Hz}$ for a quantum limited cavity (tweezer) detection. The curve labeled by 2 represents an extreme scenario where the dominating effect is the thermal noise at $300 \mathrm{mK}$ and $P=10^{-13} \mathrm{mbar}$. This might be in principle achieved with a SQUID detection and ultranarrow bandwidth $\approx 10^{-5} \mathrm{~Hz}$, and provided that electronics noise is suppressed to negligible values.

solutions require a substantial progress beyond current performance, and the solution of a lot of technical issues. In the following we optimistically assume a best scenario for all three cases, which leads to the bandwidth vs detectable $\lambda$ plots in the previous section.

By comparing Figs. 6, 9, and 12 we draw the following conclusions.

(1) Even if detecting exceedingly low forces due to CSL requires extremely low power, compared to standard optomechanical experiments, optical readout allows a much more sensitive position measurement compared to SQUIDs, which translates into a larger bandwidth, i.e., speed of measurement. For a continuous monitoring, this becomes a crucial aspect if the trap features long-term instabilities or drifts.

(2) The cavity performs better than the tweezer especially for very low values of lambda, down to $10^{-12} \mathrm{~Hz}$, however it presents more technical limitations and less flexibility. The tweezer approach is more flexible and can be used, for instance, on a wider range of power. In both situations, technical noise is hardly avoidable, in particular laser frequency noise in the cavity and detector dark noise in the tweezer. However, in the ultralow power limit the cavity performs better.

(3) A SQUID approach is clearly unsuitable under the bandwidth criterion. However, it would present some advantages. First, the power dissipated in the nanoparticle by the readout would be negligible, so that we may assume the latter to thermalize to the bath temperature. Therefore, for a given gas pressure the force noise is in general lower than in the optical case. A stronger bound on CSL could be in principle inferred, at the cost of a much longer measurement time. Second, operation at low coupling does not present the numerous technical issues of an optomechanical coupling. Therefore, a SQUID readout would be still an option if the long-term stability of the trap is good enough to resolve a peak with a bandwidth of the order of $1 \mathrm{mHz}$.

(4) The previous considerations hold for a continuous monitoring strategy. For a stroboscopic strategy the readout requires at least two features: (a) a fast and strong position measurement in order to prepare and measure the system in short time and (b) a straightforward procedure for switching on and off the readout without perturbing the system. In this respect, while the feasibility of this scheme is still to be proven, we suggest that a SQUID readout would be unsuitable because of (a), while a tweezer implementation appears more promising than a cavity one because of (b). A more detailed treatment of a realistic implementation of stroboscopic strategy will be the subject of a future paper.

Keeping in mind these considerations, we show in Fig. 15 the CSL exclusion plot analogous to what is discussed in Fig. 1 along with two representative hypothetical bounds from a levitated nanoparticle. The two optical readouts are grouped together (curve 1) since the cavity and tweezer can theoretically reach the same sensitivity in terms of CSL rate $\lambda$, however with a different bandwidth. The SQUID detection (curve 2) can in principle outperform both optical readouts, however, as stated before, at the price of an ultranarrow detection bandwidth.

\section{ACKNOWLEDGMENTS}

The authors acknowledge support from the European Union H2020 FET project TEQ (Grant No. 766900). A.P. has received funding from the European Union's Horizon 2020 research and innovation programme under the Marie Sklodowska-Curie Grant Agreement No. 749709.
[1] G. C. Ghirardi, A. Rimini, and T. Weber, Phys. Rev. D 34, 470 (1986).

[2] G. C. Ghirardi, P. Pearle, and A. Rimini, Phys. Rev. A 42, 78 (1990).

[3] G. C. Ghirardi, R. Grassi, and F. Benatti, Found. Phys. 25, 5 (1995).

[4] A. Bassi and G. C. Ghirardi, Phys. Rep. 379, 257 (2003).

[5] A. Bassi, K. Lochan, S. Satin, T. P. Singh, and H. Ulbricht, Rev. Mod. Phys. 85, 471 (2013).
[6] R. Penrose, Gen. Relativ. Gravit. 28, 581 (1996).

[7] R. Penrose, Phil. Trans. R. Soc. A 356, 1927 (1998).

[8] L. Diosi, Phys. Rev. A 40, 1165 (1989).

[9] S. L. Adler, J. Phys. A 40, 2935 (2007).

[10] K. Hornberger, S. Gerlich, P. Haslinger, S. Nimmrichter, and M. Arndt, Rev. Mod. Phys. 84, 157 (2012).

[11] S. L. Adler and F. Ramanazoglu, J. Phys. A 40, 13395 (2007).

[12] C. Curceanu, B. C. Hiesmayr, and K. Piscicchia, J. Adv. Phys. 4, 263 (2015). 
[13] M. Bahrami, M. Paternostro, A. Bassi, and H. Ulbricht, Phys. Rev. Lett. 112, 210404 (2014).

[14] B. Collett and P. Pearle, Found. Phys. 33, 1495 (2003).

[15] S. L. Adler, J. Phys. A 38, 2729 (2005).

[16] A. Bassi, E. Ippoliti, and S. L. Adler, Phys. Rev. Lett. 94, 030401 (2005).

[17] S. Nimmrichter, K. Hornberger, and K. Hammerer, Phys. Rev. Lett. 113, 020405 (2014).

[18] L. Diosi, Phys. Rev. Lett. 114, 050403 (2015).

[19] A. Vinante, M. Bahrami, A. Bassi, O. Usenko, G. Wijts, and T. H. Oosterkamp, Phys. Rev. Lett. 116, 090402 (2016).

[20] A. Vinante, R. Mezzena, P. Falferi, M. Carlesso, and A. Bassi, Phys. Rev. Lett. 119, 110401 (2017).

[21] M. Carlesso, A. Bassi, P. Falferi, and A. Vinante, Phys. Rev. D 94, 124036 (2016).

[22] M. Armano et al., Phys. Rev. Lett. 116, 231101 (2017).

[23] M. Armano et al., Phys. Rev. Lett. 120, 061101 (2018).

[24] M. Carlesso, M. Paternostro, H. Ulbricht, A. Vinante, and A. Bassi, New J. Phys. 20, 083022 (2018).

[25] S. L. Adler and A. Vinante, Phys. Rev. A 97, 052119 (2018).

[26] M. Bilardello, S. Donadi, A. Vinante, and A. Bassi, Physica A 462, 764 (2016).

[27] T. Kovachy, J. M. Hogan, A. Sugarbaker, S. M. Dickerson, C. A. Donnelly, C. Overstreet, and M. A. Kasevich, Phys. Rev. Lett. 114, 143004 (2015).

[28] D. Goldwater, M. Paternostro, and P. F. Barker, Phys. Rev. A 94, 010104(R) (2016).

[29] J. Li, S. Zippilli, J. Zhang, and D. Vitali, Phys. Rev. A 93, 050102(R) (2016).

[30] In the following we will indicate a power spectral density with the double subscript, i.e., $S_{f f}$, and amplitude spectral density with the single subscript, i.e., $S_{f}$.

[31] G. C. Ghirardi, R. Grassi, and A. Rimini, Phys. Rev. A 42, 1057 (1990).

[32] B. Helou, B. J. J. Slagmolen, D. E. McClelland, and Y. Chen, Phys. Rev. D 95, 084054 (2017).

[33] W. Paul, Rev. Mod. Phys. 62, 531 (1990).

[34] O. Romero-Isart, L. Clemente, C. Navau, A. Sanchez, and J. I. Cirac, Phys. Rev. Lett. 109, 147205 (2012).

[35] F. Liu, K. J. Daun, D. R. Snelling, and G. J. Smallwood, Appl. Phys. B 83, 355 (2006).

[36] D. E. Chang, C. A. Regal, S. B. Papp, D. J. Wilson, J. Yeb, O. Painter, H. J. Kimble, and P. Zoller, Proc. Natl. Acad. Sci. USA 107, 1005 (2010).

[37] J. Millen, T. Deesuwan, P. Barker, and J. Anders, Nat. Nanotechnol. 9, 425 (2014).
[38] G. Gabrielse, X. Fei, L. A. Orozco, R. L. Tjoelker, J. Haas, H. Kalinowsky, T. A. Trainor, and W. Kells, Phys. Rev. Lett. 65, 1317 (1990).

[39] A. Pontin, M. Bonaldi, A. Borrielli, F. S. Cataliotti, F. Marino, G. A. Prodi, E. Serra, and F. Marin, Phys. Rev. A 89, 023848 (2014).

[40] V. B. Braginsky and F. Ya. Khalili, Quantum Measurement (Cambridge University Press, Cambridge, England, 1992).

[41] V. Jain, J. Gieseler, C. Moritz, C. Dellago, R. Quidant, and L. Novotny, Phys. Rev. Lett. 116, 243601 (2016).

[42] P. F. Barker and M. N. Shneider, Phys. Rev. A 81, 023826 (2010).

[43] T. S. Monterito, J. Millen, G. A. T. Pender, F. Marquardt, D. Chang, and P. F. Barker, New J. Phys. 15, 015001 (2013).

[44] J. Millen, P. Z. G. Fonseca, T. Mavrogordatos, T. S. Monteiro, and P. F. Barker, Phys. Rev. Lett. 114, 123602 (2015).

[45] P. Z. G. Fonseca, E. B. Aranas, J. Millen, T. S. Monteiro, and P. F. Barker, Phys. Rev. Lett. 117, 173602 (2016).

[46] N. Kiesel, F. Blaser, U. Delić, D. Grass, R. Kaltenbaek, and M. Aspelmeyer, Proc. Natl. Acad. Sci. USA 110, 14180 (2013).

[47] A. C. Pflanzer, O. Romero-Isart, and J. I. Cirac, Phys. Rev. A 86, 013802 (2012).

[48] A. Pontin, C. Biancofiore, E. Serra, A. Borrielli, F. S. Cataliotti, F. Marino, G. A. Prodi, M. Bonaldi, F. Marin, and D. Vitali, Phys. Rev. A 89, 033810 (2014).

[49] M. Schwarz, O. O. Versolato, A. Windberger, F. R. Brunner, T. Ballance, S. N. Eberle, J. Ullrich, P. O. Schmidt, A. K. Hansen, A. D. Gingell, M. Drewsen, and J. R. Crespo López-Urrutia, Rev. Sci. Instrum. 83, 083115 (2012).

[50] M. Rashid, M. Toroš, and H. Ulbricht, Quantum Meas. Quantum Metrol. 4, 17 (2017).

[51] C. Timberlake, M. Toroš, D. Hempston, G. Winstone, M. Rashid, and H. Ulbricht, Appl. Phys. Lett. 114, 023104 (2019).

[52] J. Vovrosh, M. Rashid, D. Hempston, J. Bateman, M. Paternostro, and H. Ulbricht, J. Opt. Soc. Am. B 34, 1421 (2017).

[53] D. Goldwater, B. A. Stickler, L. Martinetz, T. E. Northup, K. Hornberger, and J. Millen, Quantum Sci. Technol. 4, 024003 (2019).

[54] S. Kotler, R. W. Simmonds, D. Leibfried, and D. J. Wineland, Phys. Rev. A 95, 022327 (2017).

[55] L. Baggio, M. Bignotto, M. Bonaldi, M. Cerdonio, L. Conti, P. Falferi, N. Liguori, A. Marin, R. Mezzena, A. Ortolan, S. Poggi, G. A. Prodi, F. Salemi, G. Soranzo, L. Taffarello, G. Vedovato, A. Vinante, S. Vitale, and J. P. Zendri, Phys. Rev. Lett. 94, 241101 (2005).

[56] P. Falferi, M. Cerdonio, L. Franceschini, R. Macchietto, S. Vitale, and J.-P. Zendri, Rev. Sci. Instrum. 65, 2916 (1994). 